\title{
Aceptación y uso de herbolaria en medicina familiar
}

Genaro Armando Taddei-Bringas, M.C., ${ }^{(1)}$ Manuel Alberto Santillana-Macedo, M.C., M. en C., ${ }^{(2)}$ José Alfredo Romero-Cancio, M.C., ${ }^{(2)}$ Miguel Bernardo Romero-Téllez, M.C. ${ }^{(3)}$

Taddei-Bringas GA, Santillana-Macedo MA, Romero-Cancio JA, Romero-Téllez MB. Aceptación y uso de herbolaria en medicina familiar Salud Publica Mex 1999;41:216-220.

\begin{abstract}
Resumen
Objetivo. Explorar el grado de utilización de las plantas medicinales entre la población usuaria y el personal de salud en una unidad urbana de medicina familiar del Instituto Mexicano del Seguro Social. Material y métodos Se realizó un estudio transversal descriptivo. Se diseñó y validó un cuestionario que exploró aceptación y utilización. Se aplicó a 60 médicos familiares, a una muestra aleatoria de 130 trabajadores de la Unidad de Medicina Familiar y a otra de 264 usuarios-pacientes de la misma. El porcentaje de respuesta fue de $78 \%$. Resultados. Se encontró que $83 \%$ de los médicos aceptan la herbolaria y 75\% la utilizan como recurso terapéutico. Entre el personal de salud, la aceptación y la utilización fue de $100 \%$ y, en el caso de los usuarios, 92\% la aceptan y $90 \%$ la utilizan. Las diferencias entre grupos son significativas $(p<0.05)$ Las plantas más utilizadas fueron el go rdolobo (Gnaphalium sp.), el eucalipto (Eucalyptus, probablemente E. globulus), la hierbabuena (M entha sp.), la manzanilla (M atricaria chamomilla) y el nopal (Opuntia Sp., probablemente 0 . ficus-indica). Conclusiones Esta utilización es congruente con lo que indican informes previos en México, pero se documenta como recurso terapéutico en zonas urbanas, al que recurren médicos formados en el modelo biomédico farmacológico.
\end{abstract}

Palabras clave: medicina herbaria/utilización; medicina familiar; IMSS (MX)

\author{
Taddei-Bringas GA, Santillana-Macedo MA, \\ Romero-Cancio JA, Romero-Téllez MB. \\ Acceptance and use of therapeutic medical plants \\ in family medical care. \\ Salud Publica Mex 1999;41:216-220.
}

\begin{abstract}
A bstract
Objective To explore the degree of usage of therapeutic medical plants among the patients, physicans and health workers in a local Family Mediccal C are U nit of the Mexican Institute of Social Security (IMSS)). Material and methods. A transversal descriptive study was performed. A questionnaire focusing on two variables was designed and validated. It was applied to 60 family physicians, a randomized sample of 130 health workers and another of 264 patients of the Family Mediccal C are U nit. Response percentage was $78 \%$. Results The study found that $83 \%$ of family physicians accept the therapeutic use of herbal medicine; moreover, $75 \%$ use it as a therapeutic resource.Among health workers, acceptance and use was $100 \%$, while in patients the level of acceptance was of $92 \%$ and of use it was $90 \%$. Differences between groups are significant $(p<0.05)$. The more frequently used plants are Gordolobo (Gnaphalium sp.), Eucalyptus (Eucalyptus sp., probably E. globulus), spearmint (M entha sp.), camomile (Matricaria chamomilla) and prickly pear cladodes (the vegetative parts of the prickly pear, Opuntia sp. Probably Opuntia ficus indica). Conclusions This information agrees with previous reports about Mexico, however, in this case, data were gathered in urban areas where physicians have been trained in the biomedical paradigm of medicine.
\end{abstract}

Key words: medicine, herbal/utilization; family practice; IMSS (MX)

(1) Coordinador de Consulta Externa, Unidad de Medicina Familiar N 0. 37, Instituto Mexicano del Seguro Social (IMSS), Hermosillo, Sonora, México.

(2) Médicos Familiares, Unidad de Medicina Familiar N 0. 37, IMSS, Hermosillo, Sonora, México.

(3) Delegado Eestatal, Delegación Sonora, IMSS, México

Fecha de recibido: 20 de agosto de 1998 • Fecha de aprobado: 16 de febrero de 1999

Solicitud de sobretiros: Dr. Manuel Alberto Santillana Macedo. Av. de los Mayos 1271, Fracc. Camino Real, 83148 Hermosillo, Sonora, México. 
A partir de la Declaración de Alma Ata, la Organización Mundial de la Salud propuso apoyar la utilización tanto de los recursos tradicionales como de los propios de la medicina generada en el modelo biomédico. ${ }^{1}$ Así, hoy en día se aprecia una aceptación parcial de las medicinas alternativas por parte del modelo hegemónico médico de atención, así como un incremento en su uso por la población. , $^{2,3} \mathrm{Si}$ bien este ámbito ha sido estudiado previamente dentro de la antropología médica, ${ }^{4}$ tanto la investigación médica como algunas instituciones de salud ${ }^{5}$ han desarrollado un interés propio en zonas rurales e indígenas. Así por ejemplo, el Instituto Mexicano del Seguro Social (IMSS) realizó un estudio cuya finalidad fue difundir el conocimiento y las experiencias de los terapeutas tradicionales de México con los equipos de salud de IMSS-COPLAMAR. ${ }^{6}$

En el contexto de la región Noroeste de México, son escasos los estudios sobre herbolaria; destaca el realizado por Bañuelos con mujeres mayo del sur de Sonora, ${ }^{7}$ que documentó el uso de 92 especies vegetales para el tratamiento de 53 padecimientos. En otro estudio llevado a cabo en 1991, donde se indagó el uso de remedios tradicionales a partir de un estudio transgeneracional abuela-madre-hija, se describió el patrón de permanencia y cambio de algunos tratamientos herbolarios. ${ }^{8}$ Por otro lado, el estudio de recopilación de información de la medicina tradicional, por medio de una encuesta con muestreo aleatorio, que hiciera un grupo de investigación del IMSS durante 1987, mostró que en el estado de Sonora se usaban 102 plantas medicinales. ${ }^{9}$ En ese estudio se encontró que $78 \%$ de las plantas medicinales se emplean para la atención de cuadros de patología respiratoria y digestiva aguda, así como para problemas dérmicos, lo cual coincide con los motivos de consulta más frecuente en la Unidades de Medicina Familiar (UMF) del IMSS. En forma adicional, para ese estado se ha estudiado la caracterización botánica de la herbolaria medicinal de la región. ${ }^{10}$

Sin embargo, un aspecto que no se ha trabajado es la aceptación y el uso de la medicina tradicional, tanto de la herbolaria como de otros recursos tradicionales, por personal en las instituciones de salud, en particular en unidades médicas del régimen ordinario del IMSS en las ciudades. Es probable que el uso de recursos tradicionales, así como de "medicinas alternativas" sea mucho más frecuente y diverso en el país que lo mostrado en esta primera exploración; no obstante, se hace evidente que este proceso ni es nuevo ni se encuentra negado o cuestionado totalmente en la práctica cotidiana.

Este estudio muestra un primer acercamiento a esta situación en una UMF urbana. Los objetivos fue- ron conocer, por un lado, el nivel de aceptación hacia las plantas medicinales por parte tanto de la población usuaria como del personal de salud -médico y paramédico- en una UMF del IMSS.

Por el otro lado, se buscó explorar el grado de utilización de las plantas medicinales entre la población usuaria y el personal de salud en una UMF del IMSS, así como identificar las plantas medicinales más utilizadas.

\section{Material y métodos}

Se realizó un estudio transversal descriptivo en la UMF No.37 de la ciudad de Hermosillo, Sonora. Se diseñó un cuestionario de 11 reactivos que exploró dos variables sobre la herbolaria: la aceptación, por una parte, y el uso, por la otra. El cuestionario se validó mediante su aplicación a un grupo de tres expertos, dos médicos familiares y un antropólogo, y su aplicación, primero, a un grupo piloto de 20 usuarios. El índice de concordancia (Kappa) fue de 78\%. La encuesta se aplicó a los 60 médicos familiares de la UMF a una muestra aleatoria de 130 trabajadores no médicos de la misma (50\% del total), y a una muestra aleatoria simple de 264 derechohabientes mayores de 15 años, que acudieron a la unidad médica en una semana. La muestra se determinó con un $95 \%$ de confianza y $5 \%$ de error. El porcentaje global de respuesta fue de $87 \%$.

A todos los médicos y trabajadores encuestados se les preguntó acerca de sus datos generales y de su antigüedad laboral en el IMSS. A los derechohabientes también se les preguntó acerca de la patología que habían presentado en los últimos dos años. Se hizo un análisis estadístico comparativo entre los tres grupos por comparación de medias.

\section{Resultados}

De los médicos, $85 \%$ conocen y aceptan la herbolaria, si bien el porcentaje disminuye a $75 \%$ respecto a su utilización (figura 1). Lo anterior contrasta con los resultados del resto del personal de la UMF -quienes en su totalidad conocen, aceptan y usan recursos de la herbolaria- y con los de los usuarios derechohabientes, quienes en $92 \%$ la conocen y aceptan, y en $90 \%$ la usan. Estas diferencias son estadísticamente significativas $(p<0.05)$. En el caso de los médicos los motivos por los cuales aceptan la herbolaria (figura 2) fueron su efectividad $(45 \%)$, por ser tratamientos alternativos $(24 \%)$ y por ser de uso popular $(18 \%)$. En el caso del personal de salud de la UMF, 40\% mencionó la efectividad de la herbolaria como la principal razón de su uso. En el caso de los usuarios, en su mayoría traba- 
jadores y esposas del trabajador, $78 \%$ adujeron la efectividad de la herbolaria y $67 \%$ confiaban en la misma por la popularidad de su uso. Las diferencias entre los grupos no fueron significativas $(p>0.05)$. Para el caso particular de los médicos, se indagó acerca de los motivos de rechazo a la herbolaria; $9 \%$ señaló que se desconocían sus efectos, $7.5 \%$ dijo que su uso deterioraba la imagen del IMSS y 3\% afirmó que los remedios deben recetarse sólo si se estudió medicina.

Respecto a la herbolaria que se utiliza, se encontró que, en orden decreciente, las plantas más utilizadas fueron, gordolobo (Gnaphalium sp.), eucalipto (Eucalyptus, probablemente E. globulus), hierbabuena (Mentha sp.), manzanilla (Matricaria chamomilla) y no-

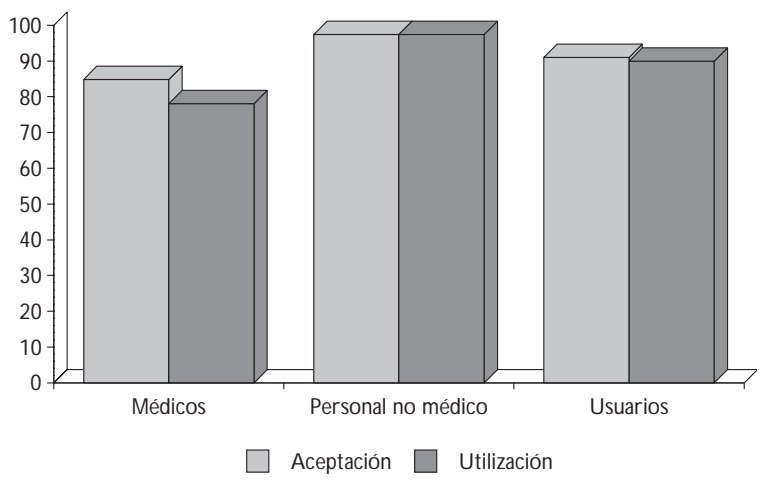

Figura 1.ACEPTACIÓN Y UTILIZACIÓN DE LA HeRbolaRia, POR EL PERSONAL MÉDICO, NO MÉDICO Y USUARIOS DE LA UMF-37. Hermosillo, Sonora, enero-marzo 1996

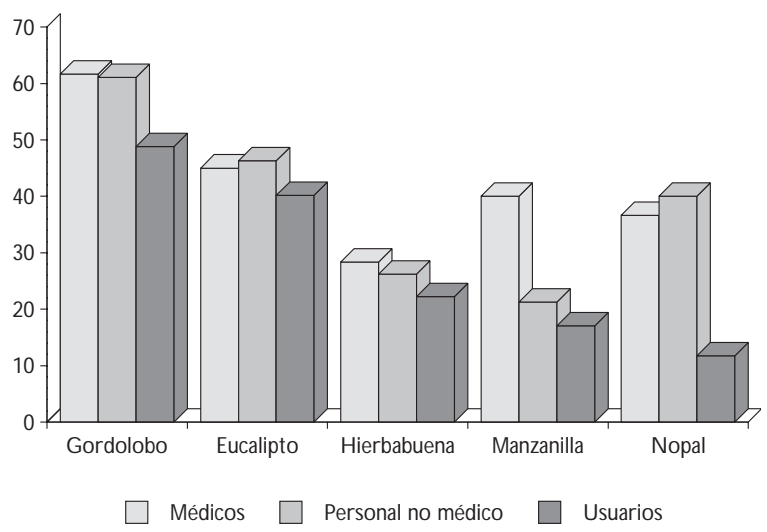

Figura 2. Plantas medicinales más utilizadas por el PERSONAL MÉDICO, NO MÉDICO Y USUARIOS DE LA UMF37. Hermosillo, Sonora, enero-marzo 1996 pal (Opuntia sp., probablemente O. ficus_indica) (figura 2). Cabe señalar que este patrón decreciente corresponde al orden de los usuarios; en el caso de los médicos se observa que la hierbabuena, con un $40 \%$, y el nopal, con un $35 \%$, se aceptan y utilizan en la consulta en cuarto y quinto lugar, respectivamente. El personal de salud también refirió utilizar más la manzanilla y el nopal, pero en orden inverso: el nopal ocupó el cuarto lugar con un $40 \%$, y la manzanilla el quinto con un $22 \%$. Para estos dos últimos remedios no se encontró una diferencia estadísticamente significativa entre grupos $(p<0.05)$.

Por otro lado, se indagó entre los usuarios acerca de los motivos por los cuales habían acudido a consulta, con el objeto de saber si había alguna relación con la herbolaria usada. Aunque $25 \%$ no contestó, se encontró que, en orden descendente, los motivos fueron: infección respiratoria aguda, 22\%; hipertensión arterial sistémica, $9.5 \%$; colitis, $8 \%$; gastritis aguda, $7.5 \%$; diabetes mellitus, $6 \%$, y cefalea, $4 \%$.

Por último, se indagó entre el personal médico de la UMF si la frecuencia del uso de la herbolaria estaba relacionada con el lugar de origen o con la antigüedad laboral. En ningún caso se encontró una diferencia significativa $(p>0.05)$.

\section{Discusión}

Este trabajo documenta la aceptación y el uso de la herbolaria en una UMF del IMSS. De los objetivos buscados en este estudio, se cumplieron los de reconocer que existe aceptación y uso por parte de médicos, personal de salud del IMSS y derechohabientes usuarios; sin embargo, fue imposible identificar a plenitud la herbolaria utilizada. Sólo se contó con la recopilación del nombre de la planta y la forma de utilizarla. Para ello, es necesario contar, además, con una muestra de cada planta utilizada y con su identificación botánica.

Si bien ya se había documentado el uso de algunos de estos recursos terapéuticos en comunidades rurales, ${ }^{11}$ lo que destaca en este estudio es su utilización por personal médico de una unidad del régimen ordinario en una zona urbana. A pesar de que no se pudo establecer una relación significativa entre el lugar de origen o la antigüedad laboral de los médicos, es interesante destacar que dos terceras partes de estos profesionales aceptan y utilizan estos remedios.

Diversos estudios en el campo de la antropología médica han documentado desde hace varios años el hecho de que existe una interrelación de los remedios de la herbolaria entre los pobladores de una localidad, los curanderos y el personal de salud institucional, médico y paramédico. ${ }^{12-14}$ Aunque en algunos de estos 
estudios se ha señalado que existe una controversia entre los recursos de la medicina alopática del modelo biomédico y la herbolaria, este estudio documenta que, de facto, existe ya dicha interrelación. Es importante reflexionar a partir de estos hallazgos, acerca del hecho de que las cifras de utilización $(83 \%$ ) y aceptación $(75 \%)$ que se encontraron corresponden a profesionales que no se habían manifestado previamente en favor de las medicinas alternativas, sino que estaban formados en el modelo biomédico y contaban con un posgrado en medicina familiar en el IMSS.

Por otra parte, es importante el hecho de que la totalidad del personal de salud de la UMF acepta y utiliza la herbolaria, sobre todo porque se ha insistido en la vinculación del trabajador no médico en muchas actividades de la promoción de la salud. ${ }^{15,16}$ En este caso en particular, el personal se vería apoyado si la institución considerara la utilización de la herbolaria como un recurso terapéutico. Así, los resultados indican la aceptación y el uso de la herbolaria a pesar de que no hay ningún programa al respecto. Sería conveniente indagar más sobre otros posibles recursos terapéuticos tales como la acupuntura, la psicoterapia y la homeopatía, que tanto la población como el personal médico y de salud del IMSS aceptan y utilizan ya.

Lo referido en este estudio acerca de la herbolaria concuerda con los informes previos de Lozoya y colaboradores ${ }^{5,6}$ así como con lo señalado regionalmente, ${ }^{7,8,10}$ lo que resulta congruente con la propuesta teórica de que el uso de la herbolaria es una práctica sociocultural ${ }^{17}$ y de que la comunidad, y ahora algunos médicos de las instituciones de salud, aceptan y utilizan este recurso aun cuando hayan sido formados teórica y prácticamente con un modelo biomédico-farmacológico de atención. . $^{18,19}$

Cabe resaltar que la posición de los autores es la de tener una actitud científica ante la herbolaria. Esto es, que se desarrolle una apertura como recurso terapéutico, ya sea con estudios que comprueben la efectividad de los procedimientos o con la implementación de estrategias de intervención que comparen su efectividad con la de los recursos farmacológicos, pero, a la vez, teniendo precaución con la promoción terapéutica por los posibles riesgos por un mal uso ${ }^{20,21} \mathrm{o}$ por la adulteración. ${ }^{22,23}$ Cabría sólo señalar que la misma actitud científica requiere ser crítico y escéptico pero abierto al conocimiento. ${ }^{24}$ Esto implica considerar que no se trata de una moda, sino de la utilización de un amplio abanico de recursos terapéuticos, entre los que se encuentra la herbolaria, pero también la homeopatía, la acupuntura, la digitopuntura, los temazcales, las diversas modalidades de psicoterapia y que, como se constata en este estudio inicial para el caso de plantas medicinales, ya los utilizan tanto la población como un porcentaje de los médicos del IMSS.

Una de las posibles observaciones de este estudio es que exploró poco el peso de las variables simbólicas; esto implica que la utilización pueda deberse a un efecto placebo.

Por otro lado, quedan elementos por conocer entre los que están la estandarización de las dosis, la homogeneización de los productos herbolarios, la adecuada concentración de los complejos activos, la identificación de efectos colaterales, los niveles de toxicidad, vida media, restricciones, etcétera. Sin embargo, se trata de un recurso terapéutico que utiliza la población para cuidar su salud, de tal manera que es necesario que lo estudie el área de la salud pública.

\section{Agradecimientos}

Los autores agradecen al doctor Alberto Búrquez M., Director del Centro de Ecología de la UNAM-Noroeste, en Hermosillo, Sonora, su valiosa colaboración en los intentos de identificación taxonómica botánica.

\section{Referencias}

1. 0 rganización Mundial de la Salud. Promoción y desarrollo de la medicina tradicional. En:Atención Primaria a la Salud. Informe de la Conferencia Internacional sobre Atención Primaria a la Salud; 1978 Alma A ta URSS.612 septiembre. Ginebra: O MS, 1978:355-367.

2. Zolla C. Terapeutas, enfermedades y recursos vegetales. México, D.F.: IN I (México Indígena), 1986:16-19.

3.Anzures y Bolaños MC. La medicina tradicional mexicana. México, D.F.: Secretaría de Educación Pública-Dirección General de Educación Indígena, 1981:157

4. LópezA ustin.Textos de medicina nahuatl.3a. edición. México,D.F.:UN AM, 1984:225.

5. Lozoya X, A guilar A, C amacho JR. Encuesta so bre el uso actual de plantas de la medicina tradicional mexicana. Rev Med Instituto Mexicano del Seguro Social 1987;(25):283-290.

6. Lozoya-Legorreta L,Velázquez-Díaz G, Flores-Alvarado A. La medicina tradicional en México. Experiencias del programa IMSS-CO PLAMAR 1982-1987. México, D.F.: IMSS, 1988:137.

7. Bañuelos N , Búrquez A. Las plantas: una estrategia de salud en la medicina doméstica mayo. Estudios Sociales 1996;12(7):163-189.

8. Santillana M. Medicina tradicional sonorense; un proceso de transición. Memorias del XIII Simposio de Historia y Antropología de Sonora, Hermosillo, 1989. Hermosillo:U niversidad de Sonora, 1990;vol.2:441-456.

9.0 suna L, Lozoya X. Plantas medicinales usadas por la medicina tradicional para el tratamiento de padecimientos gastrointestinales. Rev Med Instituto Mexicano del Seguro Social 1989;(27):305-312.

10. López-Estudillo R, Hinojosa A. Catálogo de plantas medicinales sonorenses. Hermosillo: Universidad de Sonora, 1988:143

11. Lozoya X, Zolla C. La medicina invisible. Introducción al estudio de la medicina tradicional en México. 2a. edición. México, D.F.: Folios Ediciones, 1994. 
12. Módena ME. Madres, médicos y curanderos. D iferencia cultural e identidad ideológica. México, D.F.: CIESAS, 1990:327.

13. G arcía-Jiménez S.A cerca de la medicina tradicional mexicana. C hilpancingo: Universidad Autónoma de Guerrero, 1994:120.

14. Sepúlveda J, coord. La salud de los pueblos indígenas. México, D.F.:SSAIN I, 1993.

15. Cornwall A, Jewkes R. W hat is participatory research? Soc Sci Med 1995;12(41):1676-1695.

16. Aguirre-Beltrán G. Programas de salud en la situación intercultural. México, D.F.: IMSS (C ol. Salud y Seguridad Social), 1980.

17. González-Chevez L, Hersh-Martínez P. A portes para la construcción del perfil epidemiológico sociocultural de una comunidad rural. Salud Publica Mex 1992;35(4):393-402.

18. Menéndez, Eduardo. Hacia una práctica médica alternativa. H egemonía y autoatención (cuidado)en salud. México, D.F.: CIESAS-IN AH (Cuadernos de la Casa Chata), 1982:87.
19. Menéndez E. Morir de alcohol. Saber e ideología médica. México, D.F:Alianza/C N C A, 1990:277.

20. Díaz JL. Uso de las plantas medicinales de México. México, D.F.: IMEPLAM, 1976:154.

21. Montoya-C abrera MA, Escalante-G alindo P, Meckes-Fisher M, SánchezVara G, Flores-A lvarez E, Reynoso-G arcía M. Envenenamiento mortal causado por el aceite de epazote, Chenonopodium graveolens. Gac Med Mex 1996;4(132): 433-437

22. N elson L, Shin R, Hoffman R.A plastic anemia induced by an adultered herbal medication. J Clin Toxicol 1995;(33):467-470.

23. Hersch-Martínez P. La flora medicinal como mercancía. A lgunas implicaciones sociales y ambientales en su manejo desde una zona de abasto. Bol Of Inst Nal Antrop Hist Mex 1993;(35):18-29.

24. Toledo VM. La etnobotánica hoy, reversión del conocimiento, lucha indígena y proyecto nacional. Biótica 1982;7(2):141-150. 